\title{
DESARROLLO DE UN MODELO DE DISTRIBUCIÓN URBANA DE MERCANCÍAS CON PLATAFORMAS LOGÍSTICAS APLICADO A LA CIUDAD
}

\author{
MELISSA RIVERA ARROYAVE \\ Universidad de San Buenaventura seccional \\ Medellín \\ melis1090@hotmail.com
}

\author{
VERÓNICA VELASCO NARANJO \\ Universidad de San Buenaventura \\ seccional Medellín \\ veronic105@yahoo.com
}

(Tipo de Artículo: Reflexión. Recibido el 20/05/2014. Aprobado el 25/06/2014)

\section{RESUMEN}

El transporte de mercancías es uno de los eslabones principales dentro de una cadena de suministro, por lo que es importante tener una buena logística de distribución que permita tener un transporte de mercancía lo más eficiente posible, por esto se plantea el desarrollo de un modelo matemático de distribución y asignación basado en plataformas logísticas; este modelo se realizó con base en el Problema de Ruteo de Vehículo (VRP por sus siglas en inglés) utilizando un algoritmo Greedy el cual se corrió en Java; al final se dará a conocer la simulación de un conjunto de puntos.

\section{Palabras clave}

Algoritmo Greedy, Asignación, Condiciones Urbanas, Distribución Urbana, Plataformas Logísticas, Problema de Ruteo de Vehículo.

\section{DEVELOPMENT OF A MODEL OF GOODS' URBAN DISTRIBUTION WITH LOGISTIC PLATFORMS APPLIED TO THE CITY}

\section{ABSTRACT \\ Goods transport is one of the main links in a supply chain, therefore is very important having a good distribution logistics allowing transportation of goods as efficiently as possible, for this reason is proposed the development of a mathematical model for distribution and allocation based on logistic platforms, this model has been made based on the Vehicle Routing Problem (VRP) using a Greedy algorithm which was executed in Java. \\ Keywords \\ Greedy Algorithm, Allocation, Urban conditions, Urban distribution, Logistic Platforms, Vehicle Routing Problem. \\ DÉVELOPPEMENT D'UN MODĖLE DE DISTRIBUTION URBAINE DES MARCHANDISES EN UTILISANT PLATEFORMES LOGISTIQUES APPLIQUÉES AUX VILLES}

\begin{abstract}
Résumé.
Le transport des marchandises est un des chainons principaux dans la chaine d'approvisionnement, par conséquent est très important d'avoir bon logistique de distribution qui nous permet d'avoir un transport des marchandises efficace, on propose le développement d'un modèle mathématique de distribution et répartition en se basant sur plateformes logistiques ; ce modèle est fait d'après le problème des routes de véhicules en utilisant l'algorithme Greedy sur Java.
\end{abstract}

\section{Mots-clés.}

Algorithme Greedy, Répartition, Conditions urbaines, Distribution urbaine, Plateformes logistiques, Problème des routes de véhicules. 


\section{INTRODUCCIÓN}

La logística se ha ido integrando cada día más en el mundo de la industria, convirtiéndose en una parte fundamental dentro de la planeación de las organizaciones, empresas y ciudades; es por esto que actualmente se ve en todo el mundo iniciativas de proyectos logísticos dentro de la ciudad, se busca establecer un modelo que permita integrar el transporte de mercancías con una buena distribución para establecer un orden y una armonía en la ciudad donde toda la sociedad se vea beneficiada, puesto que todo esto permite de alguna forma ser más eficiente en entregas de mercancía y más ordenados en la manera de transportarla al buscar nuevos mecanismos que no interfieran tanto si es posible en el transporte público de las personas, además de convertirse en una oportunidad para invertir en infraestructura que permita aportar al desarrollo y crecimiento de la ciudad.

Actualmente las ciudades se encuentran en constantes cambios en busca de una mejora y un crecimiento que les permita ser competitivas con las ciudades del mundo; uno de los aspectos a los que actualmente le apuntan muchas de las ciudades en el mundo es el aspecto de transporte urbano y la logística de transporte de mercancía, ya que incursionar en proyectos de logística de transporte de mercancía para una ciudad implica invertir en infraestructura, mejorar vías y adquirir nuevas tecnologías, ofreciendo a la ciudad comodidades en el transito vial, y permitiéndole crecer organizacionalmente a las empresas que se encuentren ubicadas dentro de la ciudad. Una de las opciones que tienen las ciudades es instalar plataformas logísticas que estén integradas con modelos de asignación y distribución lo que le permitirá a las ciudades mejorar el transporte de mercancía. En este proyecto como tal se propuso un modelo de asignación y distribución basado en el Problema de Ruteo de Vehículo y el Algoritmo de Greedy

\section{LOGÍSTICA}

La logística consiste en un conjunto de actividades y operaciones como son el envío, la recepción, el empaque $u$ organización de los productos que se manejan dentro de la empresa.

Durante todos estos años siempre se ha tenido presente el término logística puesto que muchos autores se han referido a este término como una pieza fundamental para el buen funcionamiento de una empresa o proceso.

\subsection{Logística urbana y distribución de mercancías.}

La logística urbana envuelve diferentes temas, tales como, tarifas del transporte, disponibilidad de equipos, transporte y lugares de almacenamiento, avances tecnológicos, normatividades o leyes, entre otros [2]; todo estas actividades son igual de importantes en el tema de logística por lo que se debe procurar mantener un equilibrio entre cada una de ellas para poder lograr tener una distribución de mercancía lo más óptima posible.

Hoy en día se puede encontrar diferentes opiniones que en su momento (algunos más recientes que otros) escribieron ciertos autores acerca de la logística urbana como lo son el caso de Taniguchi, quien dijo que la logística urbana era el proceso para optimizar por completo las actividades logísticas y de transporte de las empresas privadas en las zonas urbanas, apoyándose en sistemas avanzados de información [16].

Valles identifica diferentes sistemas que se pueden encontrar dentro de la logística urbana como son: sistemas subterráneos para el transporte de mercancías, sistemas inteligentes sobre viajes, terminales logísticas como centros de distribución y transbordo [17].

Una opción entonces de mejorar y manejar en conjunto todas las actividades que engloba la logística de distribución es la utilización de plataformas logísticas, las cuales se están volviendo una buena opción en algunas partes del mundo.

\subsection{Plataformas logísticas}

Las plataformas logísticas son zonas ubicadas estratégicamente en la red urbana de la ciudad y allí se ejercen actividades relativas al transporte, empaque, distribución y almacenamiento de carga para tránsito nacional o internacional [12].

Estas plataformas son áreas especializadas que garantizan un transporte seguro y una entrega a tiempo de la mercancía a las empresas, por lo que un buen manejo de estas plataformas genera un desarrollo industrial y afianza relaciones comerciales.

Las plataformas logísticas pueden presentarse de un nivel o dos niveles; la de un nivel está constituida por una sola estación, y consiste en llevar la mercancía a la ciudad en camiones de mediana carga, se debe descargar la mercancía en la plataforma y allí se encargan de empacar la mercancía en los camiones medianos; por otra parte en las plataformas de dos niveles se realizan dos transbordos para que la mercancía llegue en vehículos aún más pequeños, garantizando una fácil y rápida movilización dentro de la ciudad (Ver figura 1 y 2 )

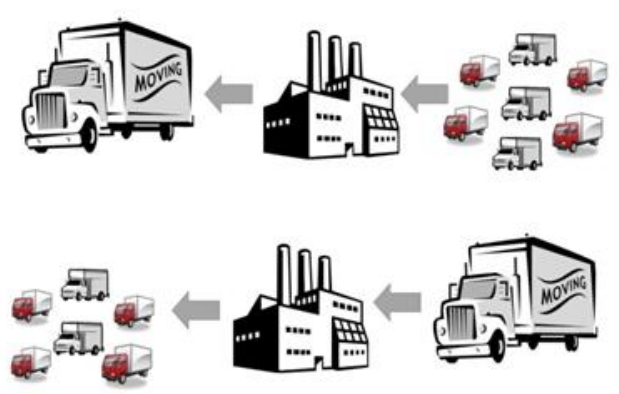

Fig. 1. Plataforma Logística de un nivel 


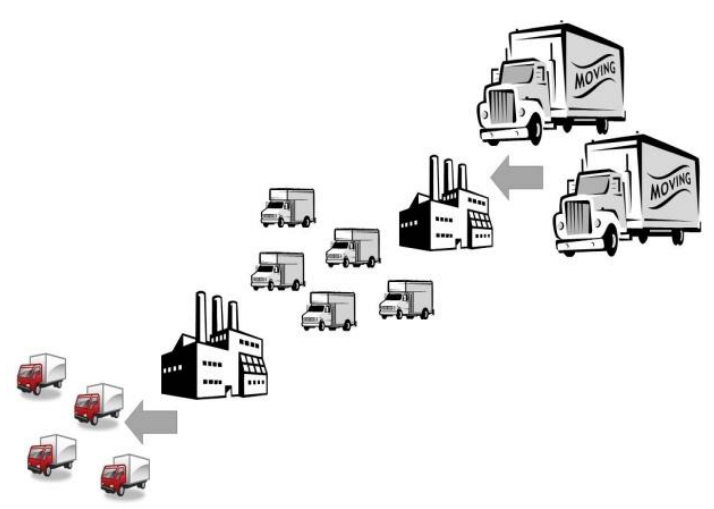

Fig. 2. Plataforma Logística de dos niveles

Como se mencionó anteriormente en las plataformas logísticas se almacena la mercancía que posteriormente deberá ser entregada en los diferentes lugares de la ciudad, por lo tanto en estas plataformas también se encargan de controlar y planear las mejores rutas para distribuir la mercancía, esto normalmente se realiza zonificando la ciudad y creando clusters (conjunto de clientes cercanos que se visitan realizando la misma ruta) cercanos buscando siempre una operación eficiente y que optimice tiempo y costos; debido a estas actividades es probable que se presenten algunos problemas, entre ellos el ruteo.

\subsection{Problemática del ruteo y asignación}

La actividad de planear las rutas para la distribución de mercancías envuelve diferentes factores que hay que tener en cuenta en el momento de decidir los clusters o el orden de la ruta, tomando como base algunas opiniones de Olivera, se concluye lo siguiente:

La demanda de cada cliente por ejemplo es un factor que se debe tener muy presente ya que el objetivo es satisfacer esa demanda entonces en el momento de asignar las rutas se debe mirar la capacidad de los vehículos y cuantos clientes se pueden visitar con el mismo vehículo según su demanda, la idea sería atender el mayor número de clientes cercanos pero cumpliendo siempre con la necesidad total del cliente, aquí se podrían presentar problemas si en la plataformas no se cuenta con suficientes vehículos para sobrellevar estas situaciones.

La ubicación y las distancias entre clientes son otros aspectos que se deben tener en cuenta para lograr optimizar tiempos y costos, además es importante conocer la variabilidad de la demanda de los clientes para tener una mejor programación de los vehículos y formar las rutas de la mejor manera posible, acá se debe tener en cuenta un aspecto muy importante que es la asignación de rutas a cada vehículo; es muy importante que exista hasta donde sea posible una buena coherencia entre la ruta y la asignación de ésta teniendo siempre en cuenta la demanda y los vehículos disponibles.

Los vehículos son otro factor importante en la operación de la distribución puesto que es el medio que se utiliza para transportar la mercancía, los aspectos que están relacionados entonces con los vehículos serían la capacidad y el peso máximo que soportan, además se debe tener conocimiento del lugar donde esté ubicado el cliente y el tipo de restricciones que hay para acceder a cada lugar; en este caso se debe tener en cuenta los costos en los que incurre el vehículo y procurar librarlos con las operaciones realizadas en las plataformas [11].

Actualmente existen modelos matemáticos que facilitan un poco esta distribución de mercancía, ofreciendo así varias posibilidades según el modelo operativo que se desee utilizar en las plataformas logísticas. Además también se cuenta con métodos para apoyar el aspecto o problemática de asignación, permitiendo mejorar la logística de distribución de las plataformas o cualquier centro de distribución que tenga la posibilidad de utilizar adecuadamente estas herramientas.

\subsection{Modelos matemáticos}

Dentro de los modelos matemáticos que sirven como apoyo para la distribución de mercancías se encuentran:

\subsubsection{Problema del Agente Viajero (TSP)}

EI TSP consiste en la programación de visitas a sus clientes que desea realizar un agente viajero, viajando lo mínimo posible; por lo que se debe determinar una ruta que minimice la distancia total (o el tiempo o el costo) necesaria para visitar todas las zonas en la ciudad [15]. Este modelo cuenta con un vehículo el cual debe realizar todas las visitas a los clientes en una misma ruta, con un mínimo costo y sin tener en cuenta la demanda de los clientes.

Por otra parte existe una generalidad de este modelo la cual es el m-TSP, donde se cuenta con $m$ vehículos y un depósito, aquí se construyen m rutas, una para cada vehículo y se garantiza visitar a cada cliente una vez por uno de los vehículos, se debe tener en cuenta que los vehículos inician en la plataforma o depósito y finalizan su recorrido cuando lleguen nuevamente a la plataforma.

\subsubsection{VRP (Vehicle Routing Problems)}

Este modelo es uno de los más conocidos, aquí se supone la existencia de un depósito central, donde se encuentran cierta cantidad de vehículos los cuales deben atender ciertos clientes distribuidos en la ciudad, buscando lograr el principal objetivo que es minimizar costos por medio de la ruta más óptima que empiece y finalice en el depósito [8]. Aquí los clientes tienen unas demandas establecidas y los vehículos cuentan con una capacidad de carga específica. En las figura 3 y la figura 4 se pueden observar de manera general como funciona este modelo; en la figura 3 se pueden observar todas las posibles rutas y la ubicación de los clientes para distribuir las mercancías y en la figura 4 se observa la aplicación del modelo VRP, se puede 
observar la selección de la ruta para cada vehículo, teniendo en cuenta la cercanía entre los puntos que en este caso serían los clientes.

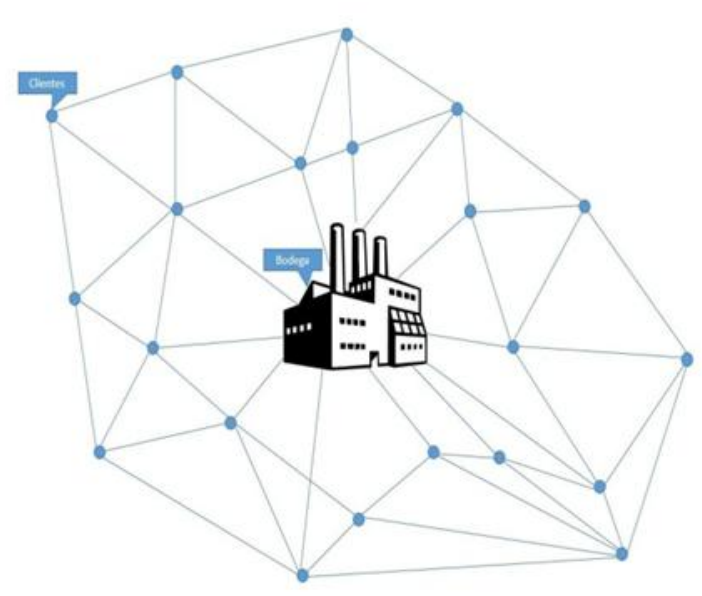

Fig. 3. Rutas de distribución

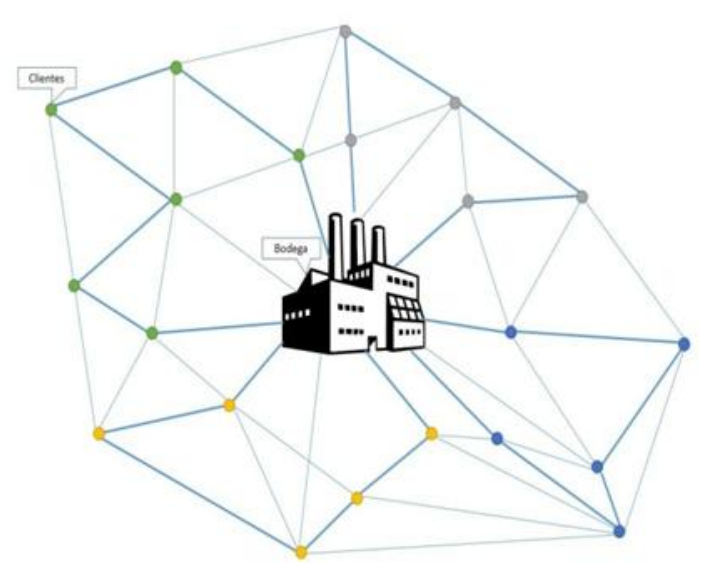

Fig. 4. Sistema de distribución VRP

Para llevar a cabo estos modelos se utilizan algoritmos que permitan simular y establecer correctamente el modelo que se quiere aplicar en la plataforma.

\subsection{Algoritmos Matemáticos}

Para solucionar algún problema de distribución se puede utilizar entonces un algoritmo matemático, estos algoritmos se utilizan según la necesidad y el procedimiento que se lleve a cabo; los tres tipos de algoritmos más conocidos son los exactos, heurísticos y metaheuristicos [14], entre estos tres grupos de algoritmos se pueden encontrar diferentes técnicas 0 formas de aplicar los algoritmos (Ver Tabla 1).

Algoritmos Exactos: estos algoritmos realizan una búsqueda detallada para encontrar dentro de varias soluciones posibles, la mejor.

Algoritmos Heurísticos: son algoritmos que utilizan varios ensayos o repeticiones antes de llegar a la solución.
Algoritmos Metaheurísticos: son algoritmos que encuentran soluciones aproximadas a los problemas, basándose o utilizando heurísticas existentes [14].

Tabla 1. Algoritmos matemáticos

\begin{tabular}{|c|c|c|}
\hline Algoritmos & Clasificación & Descripción \\
\hline $\begin{array}{c}\text { Ramificación } \\
\text { y } \\
\text { Acotamiento }\end{array}$ & Exacto & $\begin{array}{l}\text { Este algoritmo mantiene } \\
\text { un valor superior e } \\
\text { inferior del valor óptimo } \\
\text { de la función objetivo. El } \\
\text { algoritmo consiste en } \\
\text { formar ramificaciones } \\
\text { donde las ramas finales } \\
\text { tendrán todas las } \\
\text { soluciones factibles del } \\
\text { problema, si un nodo del } \\
\text { árbol no necesita más } \\
\text { ramificaciones porque ya } \\
\text { no es una solución } \\
\text { factible se poda o acota } \\
\text { [3]. }\end{array}$ \\
\hline $\begin{array}{c}\text { Ahorros de } \\
\text { Clarke and } \\
\text { Wright }\end{array}$ & Heurística & $\begin{array}{l}\text { La idea básicamente es } \\
\text { fusionar dos rutas en una } \\
\text { sola para generar un } \\
\text { ahorro en la distancia } \\
\text { recorrida, se debe mirar } \\
\text { cuales son las fusiones } \\
\text { factibles y con cuales se } \\
\text { generan mayor ahorro } \\
\text { terminando cuando no se } \\
\text { tengan rutas para } \\
\text { fusionar [3]. }\end{array}$ \\
\hline Greedy & Heurística & $\begin{array}{l}\text { Usado para solucionar } \\
\text { algunos problemas } \\
\text { conocidos, entre ellos, el } \\
\text { problema del agente } \\
\text { viajero, cuya relación es } \\
\text { referente a la ruta más } \\
\text { corta o más económica } \\
\text { posible, aunque parece } \\
\text { mostrar soluciones } \\
\text { óptimas, no siempre la } \\
\text { solución de este } \\
\text { algoritmo responde a la } \\
\text { mejor posible, por esta } \\
\text { razón es necesario } \\
\text { modificar algunos } \\
\text { detalles en la } \\
\text { programación. }\end{array}$ \\
\hline $\begin{array}{l}\text { Búsqueda } \\
\text { Tabú }\end{array}$ & Metaheurística & $\begin{array}{l}\text { Es un método que } \\
\text { genera una memoria } \\
\text { donde registra todas las } \\
\text { posibles soluciones, } \\
\text { obligando a realizar un } \\
\text { recorrido para ir } \\
\text { encontrando la mejor } \\
\text { solución de la función } \\
\text { objetivo [9]. }\end{array}$ \\
\hline Genéticos & Metaheurística & $\begin{array}{l}\text { Métodos adaptativos que } \\
\text { pueden usarse para } \\
\text { resolver problemas de } \\
\text { búsqueda y optimización, } \\
\text { se basan en seleccionar }\end{array}$ \\
\hline
\end{tabular}




\begin{tabular}{|c|c|c|}
\hline Algoritmos & Clasificación & Descripción \\
\hline & & $\begin{array}{l}\text { las soluciones del } \\
\text { problema que funcionan } \\
\text { para poder mejorarlas, lo } \\
\text { que se logra con los } \\
\text { algoritmos genéticos es } \\
\text { combinar o cruzar dos } \\
\text { soluciones formando una } \\
\text { nueva y mejor solución, } \\
\text { a la hora de realizar el } \\
\text { algoritmo se especifica el } \\
\text { número de iteraciones } \\
\text { que se van a realizar [3]. }\end{array}$ \\
\hline $\begin{array}{c}\text { Colonia de } \\
\text { Hormigas }\end{array}$ & Metaheurística & $\begin{array}{l}\text { Este Algoritmo se basa } \\
\text { en el comportamiento de } \\
\text { las hormigas en sus } \\
\text { colonias; consiste en que } \\
\text { varias hormigas exploren } \\
\text { distintas direcciones o } \\
\text { puntos de posibles } \\
\text { soluciones, dejando un } \\
\text { rastro de feromona que } \\
\text { indica a las demás } \\
\text { hormigas las posibles } \\
\text { mejores rutas, así hasta } \\
\text { encontrar la ruta más } \\
\text { óptima; este algoritmo } \\
\text { también busca minimizar } \\
\text { el número de vehículos y } \\
\text { el tiempo de entrega [9]. }\end{array}$ \\
\hline
\end{tabular}

\subsection{Método de asignación}

A continuación se darán a conocer algunos de los métodos de asignación mencionados por Olivera, los cuales serían útiles para la asignación en la operación logística de distribución de mercancías.

\subsubsection{Heurística de barrido}

En donde los grupos de clientes asignados se forman girando una semirrecta con origen en la plataforma logística e incorporando cada cliente "barrido" por dicha semirrecta hasta que se viole la restricción de la capacidad de los camiones [11].

\subsubsection{Heurística de Asignación Generalizada de Fisher y Jaikumar}

Proponen generar los clusters resolviendo un Problema de Asignación Generalizada (GAP) sobre los clientes. Primero se fijan $\mathrm{K}$ clientes semilla sk con

$\mathrm{k}=1, \ldots, \mathrm{K}$, sobre la base de los cuales se construirán los clusters. En una segunda fase, se decide qué clientes asignar a cada uno de los clusters de modo de no violar la capacidad del vehículo [11].

\subsubsection{Heurística de Localización de Bramel y Simchi-Levi}

En esta propuesta, los clientes semilla son determinados por el algoritmo resolviendo un Problema de Localización de Concentradores con Capacidades (CCLP). El CCLP se describe a continuación. Se dispone de $\mathrm{m}$ posibles ubicaciones para concentradores de capacidad Qj $(j=1, \ldots \mathrm{m})$ y $\mathrm{n}$ terminales, cada uno de los cuales utiliza wi $(i=1, \ldots n)$ de la capacidad del concentrador al que se conecta. El costo por ubicar un concentrador en la ubicación j es fj $y$ el costo de conectar el terminal i al concentrador $j$ es C'ij. El CCLP consiste en decidir cuales concentradores colocar y qué terminales conectar a cada concentrador de modo que cada terminal se conecte con exactamente un concentrador, se satisfagan las restricciones de capacidad y se minimicen los costos [11].

\section{CASOS RELACIONADOS}

Actualmente en muchos países y ciudades se están preocupando por mejorar la logística de transporte de carga, por lo que están incursionando en nuevos proyectos o simplemente están en el proceso de investigación para dar a conocer en un futuro posibles propuestas que sirvan como iniciativa para otras ciudades.

\subsection{Casos del exterior}

CIVITAS es un programa europeo con el cual se busca implementar un transporte limpio, sostenible y eficiente. Dentro de las medidas que proponen están:

- Cooperación de transportistas y operadores en los repartos y la distribución, con el fin de minimizar los desplazamientos, además de compartir cargamentos para maximizar la capacidad de los vehículos.

- Creación de plataformas logísticas que relacionen comercio, industria, servicios, compañías de transporte, etc.

- Gestionar espacios de carga y descarga e implementar tecnologías con sistemas de transporte inteligentes [5].

En Chile encontramos un caso de una plataforma logística en la región Bio Bio, esta plataforma surgió por la necesidad de rediseñar las redes de distribución de mercancía de este país. Esta plataforma cuenta con conexión directa a los sistemas de transporte como el aeropuerto Carriel Sur, terminales marítimas, autopista y red ferroviaria; además cuentan con un gran espacio físico, instalaciones aduaneras y administrativas, sistemas de seguridad y gran facilidad para el manejo de carga [1].

Por otro lado, Londres tiene el reto de lograr un sistema de distribución eficiente y sostenible de bienes y servicios. Para esto, el alcalde a principios del 2002 creó la Asociación de Distribución Sostenible de Londres (LSDP), la cual se basa en la construcción de una plataforma logística que garantice una buena distribución de mercancías, y al mismo tiempo se dé una disminución en la congestión vehicular y en la 
contaminación ambiental, además de fomentar un transporte más sostenible como el ferrocarril [4].

Otro caso es el de Sevilla, España, el cual está detallado en la síntesis de una conferencia sobre logística, donde se expone que el transporte de mercancía presenta algunos problemas como: colapso de las zonas de carga y descarga, incompatibilidad de los horarios de apertura de los comercios con los horarios definidos para el acceso de vehículos de mercancías al centro histórico, mala accesibilidad de la zona, entre otros. Por lo que algunas de las alternativas propuestas para dar solución a los problemas de distribución de esa ciudad fueron [10]:

- Repartos nocturnos: la idea es realizar el transporte de mercancías durante la noche para evitar el tráfico de la mañana.

- Lanzaderas: serian "zonas lanzaderas" ubicadas en el centro de la ciudad donde se ubicarían los camiones con la mercancía, la cual se pasaría a vehículos más pequeños para poder realizar la entrega final. Cumpliendo el papel de una plataforma logística.

- Reparto conjunto: se busca que los transportistas trabajen conjuntamente compartiendo rutas $y$ carga.

- Sistemas de información en tiempo real: consiste en brindarle información actualizada sobre el estado del tráfico a los transportadores y las empresas [10].

\subsection{Situación en Colombia}

Dentro del Informe de Competitividad 2012-2013 se encuentra que nuestro país en el aspecto de infraestructura general aparece en el puesto 117 entre 148 países, en el aspecto de desempeño logístico según el Informe está mejorando poco a poco ya que paso de estar ubicado en el puesto 82 (entre 150 países) en el año 2007 al puesto 64 (entre 155 países) en el año 2012 [6], [7].

Según este Informe de Competitividad dentro de las variables que afectan la logística del país se encuentran: la infraestructura, los atrasos en la implementación de proyectos (la mayoría de proyectos no alcanzan a desarrollarse ni en un $50 \%$ durante el tiempo estimado desde un principio), altos costos del transporte, la informalidad en las compañías de transporte y su falta de esquemas de gestión, la falta de plataformas logísticas, tiempos muertos en los puertos, falta de operadores logísticos, entre otros [6], [7].

Actualmente en las ciudades del país se pueden encontrar varios operadores logísticos o centros de distribución de carácter público o privado. Dentro del sector privado están Coordinadora, Botero Soto, Servientrega, entre otras empresas, las cuales se encargan básicamente de transportar la mercancía de las empresas o personas naturales y entregarla en el menor tiempo posible garantizando calidad $y$ puntualidad en sus entregas.

Por parte del estado se cuenta con los aeropuertos donde se destaca el aeropuerto de Rionegro José María Córdova ya que es considerado uno de los aeropuertos que mayor carga moviliza a nivel nacional e internacional.

\section{DISEÑO MODELO}

Para el planteamiento del modelo se deben tener en cuenta ciertos aspectos como las condiciones a las cuales se desea adaptar el modelo, tener claro que es lo que se pretende solucionar o mejorar, que restricciones se puede presentar, entre otros.

Se propone la ubicación de una plataforma logística, la idea es que esta plataforma esté ubicada en el lugar donde transcurra y entre más mercancía a la ciudad, según sea el movimiento de mercancías en la ciudad se decide si se ubican una, dos o más plataformas, esto depende de la necesidad de cada ciudad. Estas plataformas servirán para controlar el ingreso y salida de los productos que allí descarguen, será el punto de salida de los vehículos al centro de la ciudad y de llegada después de entregar la mercancía a cada cliente.

Esta plataforma puede ser utilizada por los diferentes operadores logísticos ubicados en la ciudad, esto permitirá estructurar un proyecto logístico sólido que beneficie a la ciudad, los clientes y las empresas, puesto que se establecerá un mejor seguimiento de la distribución de mercancías, además de apuntarle a una reducción de costos de servicio y a una operación cada vez más eficiente.

Tener en cuenta cuales son las condiciones urbanas de una ciudad es muy importante para saber cuáles pueden ser las acciones a realizar; es por esto que brevemente se mencionaran algunas condiciones que se pueden encontrar en las ciudades.

\subsection{Condiciones Urbanas}

Cada ciudad tiene diferentes condiciones de vía y terreno, básicamente se puede encontrar ciudades con topografía irregular y pendiente lo cual dificulta el transporte; se tienen tres posibles tipos de terreno de la vía en una ciudad que son ondulado, plano o montañoso, esto dependerá de la ubicación geográfica de la ciudad. Estas condiciones se deben tener en cuenta en el momento de implementar un modelo logístico para saber cómo se va operar el transporte de la mercancía.

También es muy importante identificar cuáles son los principales corredores viales de la ciudad y con cuantos cuenta, además de tener presente cuales serían las 
posibles vías alternas que se puedan utilizar cuando ocurra algún percance.

Normatividad; Colombia tiene establecido algunas restricciones para vehículos de carga con una capacidad de 3,4 toneladas en adelante. En todas las ciudades se manejan leyes para el transporte de mercancía donde básicamente se tratan de las restricciones de zonas de parqueo y horarios para carga o descarga de la mercancía, es importante entonces identificar estas leyes según la ciudad para planificar adecuadamente la distribución de la mercancía.

Medio Ambiente; el alto flujo de vehículos y el mal estado de algunos que todavía siguen operando, no solo ocasiona una gran congestión vehicular, sino que está causando contaminación ambiental: por lo que es importante tener siempre presente opciones que sean amigables con el medio ambiente.

\subsection{Modelo Matemático}

El modelo matemático está basado en el modelo VRP, el cual se dará a conocer a continuación, donde la formula plantea minimizar los costos teniendo en cuenta algunas restricciones [13].

$$
M I N \sum_{k \in K} \sum_{(\mathbb{i}(j)) \in A} C_{i j}^{k} X_{i j}^{k}
$$

Tabla 2. Restricciones del modelo

\begin{tabular}{|c|c|c|}
\hline Restricción & Complemento & Explicación \\
\hline$\sum_{k \in \mathbb{K}} \sum_{j:(i, j) \in A} x_{i j}^{k}=1$ & $\forall i \in N$ & $\begin{array}{l}\text { Garantiza que } \\
\text { todos los puntos } \\
\text { sean visitados }\end{array}$ \\
\hline$\sum_{f:(0, j) \in A} x_{0 j}^{k}=1$ & $\forall k \in K$ & \multirow{2}{*}{$\begin{array}{l}\text { Asegura que todos } \\
\text { los vehículos } \\
\text { empiecen en el } \\
\text { depósito y se } \\
\text { devuelvan al } \\
\text { finalizar su ruta. }\end{array}$} \\
\hline 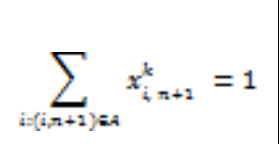 & $\forall k \in K$ & \\
\hline 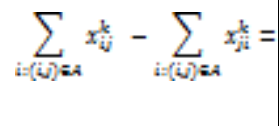 & $\forall j \in N, k \in K$ & $\begin{array}{l}\text { Conservación del } \\
\text { flujo, que no se } \\
\text { devuelva. }\end{array}$ \\
\hline $\begin{array}{c}x_{i j i j}^{k}=1 \Rightarrow B_{j}^{k} \geq \\
B_{i}^{k}+d_{i}+t_{i j}^{k}\end{array}$ & $\forall(i, j) \in A_{2} k \in K$ & $\begin{array}{l}\text { Se incluyen } \\
\text { variables de tiempo } \\
\text { para eliminar } \\
\text { subtours, dado que } \\
\left(t_{i j}^{k R}+d_{i}\right)>0 \text { para } \\
\text { todo } \\
\left(i_{v} j\right) \in A_{v} k \in K .\end{array}$ \\
\hline
\end{tabular}

\begin{tabular}{|c|c|c|}
\hline Restricción & Complemento & Explicación \\
\hline $\begin{array}{c}x_{i j i}^{k}=1 \Rightarrow Q_{j}^{k}= \\
Q_{i}^{k}+q_{i}\end{array}$ & $\forall(i, j) \in A_{a} k \in K$ & \multirow{2}{*}{$\begin{array}{l}\text { Asegura que los } \\
\text { vehículos no } \\
\text { excedan su } \\
\text { capacidad de carga } \\
\text { durante su } \\
\text { recorrido. La carga } \\
\text { inicial del vehículo } \\
\text { se ajusta a lo } \\
\text { debidamente } \\
\text { entregado, o todo } \\
q_{j} \geq 0 \text {. }\end{array}$} \\
\hline $\max \left\{o_{1} q_{i}\right\} \leq Q_{i}^{k} \leq$ & ninlet, elvithel $K$ & \\
\hline$x_{i j}^{k} \in\{0,1\}$ & $\forall(i, j) \in A_{2} k \in K$ & \\
\hline
\end{tabular}

$\mathrm{N}$ : conjunto de clientes a visitar

$\mathrm{n}$ : número de puntos de entrega

$A$ : conjuntos de $\operatorname{arcos}(i, j)$ donde $i, j \in n$

$\mathrm{K}$ : conjunto de vehículos

qi: demanda del punto $i$, el depósito inicial es 0 y el final es $n+1$, la demanda es cero $q 0=q n+1=0$

ei: primer hora para empezar el servicio en el punto i

li: hora límite para iniciar el servicio en el punto i

di: duración del servicio en el punto i

CijK: costo de atravesar el arco de i al j (i, j) con el vehículo $\mathrm{k}$

tijK: tiempo de viaje del punto i al j con el vehículo $\mathrm{k}$

CK: capacidad del vehículo $\mathrm{k}$

Tk: duración máxima de la ruta con el vehículo $\mathrm{k}$ $x_{i j}^{k}$ : toma el valor de 1 si el vehículo k atravesó el arco (i, j) y toma el valor de 0 si no es así.

$Q_{i}^{k}:$ carga del vehículo $\mathrm{k}$ al salir vértice $\mathrm{i}$.

$B_{i}^{k}$ : Inicio del servicio del vehículo $\mathrm{k}$ en el vértice $\mathrm{i}$.

Para resolver este modelo matemático se debe acudir a alternativas de ruteo y asignación, el método propuesto para la solución de este modelo es conocido como Rutear Primero-Asignar Después el cual se explicara más adelante.

\subsection{Plataformas, ruteo y asignación}

Conociendo el modelo matemático y las condiciones urbanas en general de la ciudad, se proponen entonces algunas estrategias que serán útiles para la asignación, el ruteo y las operaciones dentro de las plataformas.

Problemas de vías en el centro de las ciudades como calles estrechas o grandes pendientes en algunos barrios, se pueden mitigar asignando vehículos pequeños que tengan un fácil acceso. Para evitar inconformidad a los transeúntes en el momento de descargue de mercancía en las zonas amarillas se sugiere acordar con los clientes horarios nocturnos (esto es recomendable en zonas que estén muy transitadas en el día).

En el momento que las ciudades cuenten con las plataformas logísticas es importante tener en cuenta 
que deben buscar apoyarse en las TIC's, para que el funcionamiento sea más eficiente; se podría entonces utilizar aplicaciones móviles para el control de los vehículos, GPS y diferentes rastreadores que permitan saber con exactitud la ubicación del vehículo; también sería útil la implementación de códigos de barras para el control de inventario dentro de la plataforma.

Ya para apoyar un poco la parte del ruteo se tiene un método mencionado anteriormente descrito por Olivera, conocido como "Rutear Primero - Asignar Después", en este método primero se genera una ruta que pase por todos los puntos sin tener en cuenta las restricciones del problema, y a partir de esta ruta se realiza la asignación a cada camión de acuerdo a su capacidad [11].

El método Greedy es el que se utilizó para el ruteo, con este método se pretende generar una ruta a seguir, sin importar la capacidad del vehículo (esta ruta se crea suponiendo un solo vehículo), según la teoría de la ruta más corta, para esto se desarrolla un algoritmo de programación, que arroje una matriz de costos dependiendo de los puntos que se le introduzcan. Si se conocen los puntos en el plano cartesiano, representando la ciudad, se podrá conocer los nodos los cuales se identificaran como los depósitos o clientes que se deben visitar, con esto se obtendrán las distancias y los costos de las rutas, obteniendo al final una matriz de costos.

Partiendo del punto cero que será el centro de distribución o plataforma logística, se recorrerá la matriz para elegir una buena ruta de acuerdo a los costos previamente calculados. Se generará un vector que indica el recorrido que debe seguir el vehículo y según esto se calculará el costo total del recorrido.

El pseudocódigo que se usa para resolver esto, es el siguiente:

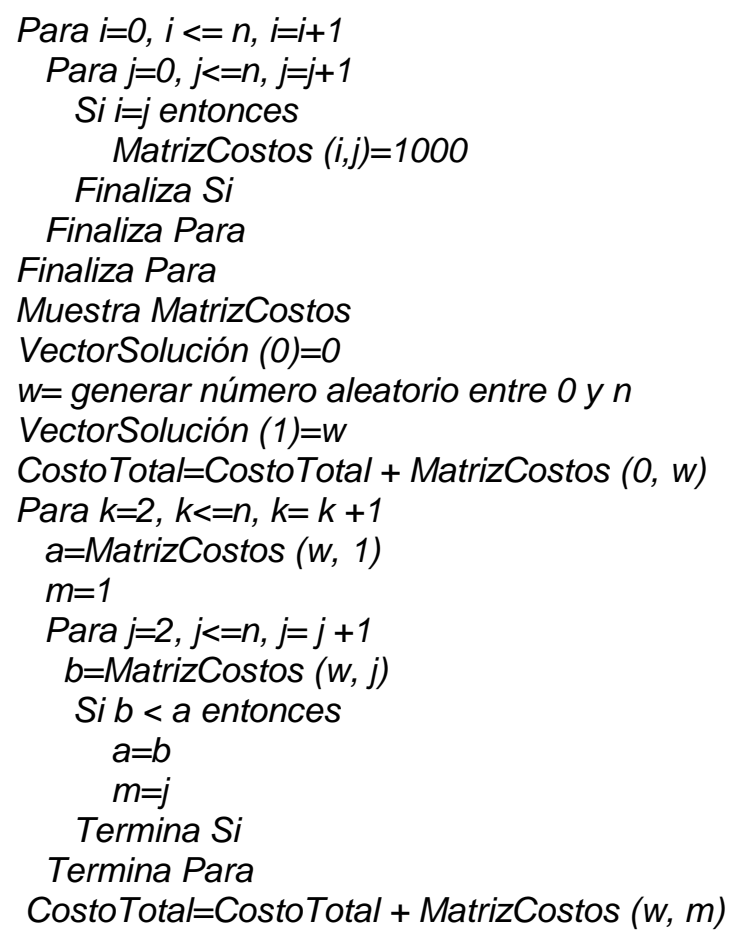

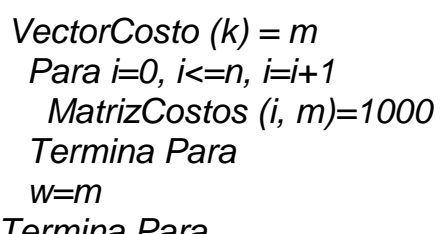

Termina Para

CostoTotal $=$ CostoTotal + MatrizCostos $(m, 0)$

Muestra VectorCosto

Muestra CostoTotal

Después de definir la ruta con ayuda del modelo y el pseudocódigo, se debe entonces continuar con la asignación, la cual se propone realizarla según el vector resultante; donde se asignará el primer camión a partir del primer cliente a visitar y así sucesivamente hasta alcanzar la capacidad del primer vehículo, luego se asignara otro vehículo continuando con el cliente correspondiente según el vector resultante del código. Al momento de asignar y seguir con la ruta estipulada se debe tener en cuenta la capacidad de los camiones, las necesidades de los clientes, cada cuanto se deben visitar, el tiempo que se demora cada vehículo para ir de un lugar a otro y la hora máxima que el cliente necesita que se le entregue la mercancía.

\section{APLICACIÓN}

Se realizó una simulación, utilizando una instancia de Capacitated VRP extraída de la página: http://www.bernabe.dorronsoro.es/vrp/; corrido en lenguaje Java utilizando Eclipse.

La instancia con la que se trabajó es de 80 Nodos, además se tienen dos Depósitos; Depósito 1 ubicado en el nodo $\{1,0\}$ y Depósito 2 ubicado en el nodo $\{90,0\}$.

En la figura 5 que aparece a continuación, se puede observar la asignación que realiza el algoritmo, esta asignación no tiene en cuenta la capacidad de los depósitos, solo utiliza las rutas y las distancias para asignar.

Los puntos verdes ilustran los dos depósitos, los nodos están divididos por color rojo y azul; donde cada conjunto de nodos del mismo color pertenecen a uno de los depósitos.

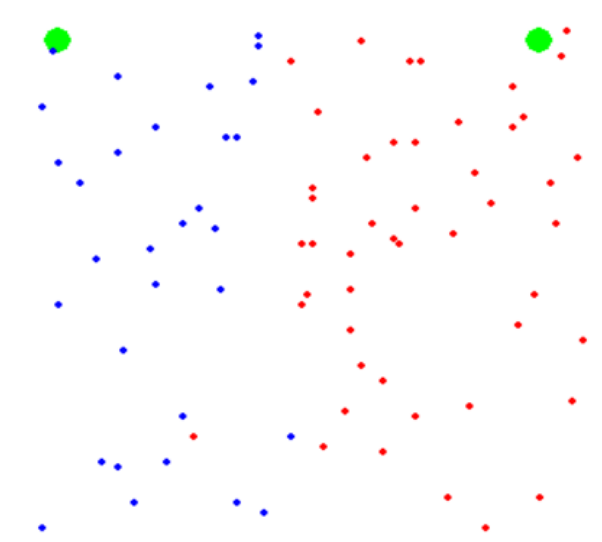

Fig. 5. Ilustración de la asignación de los Nodos 
Luego tenemos la figura 6 donde se observa la ruta que arroja el algoritmo para cada depósito.

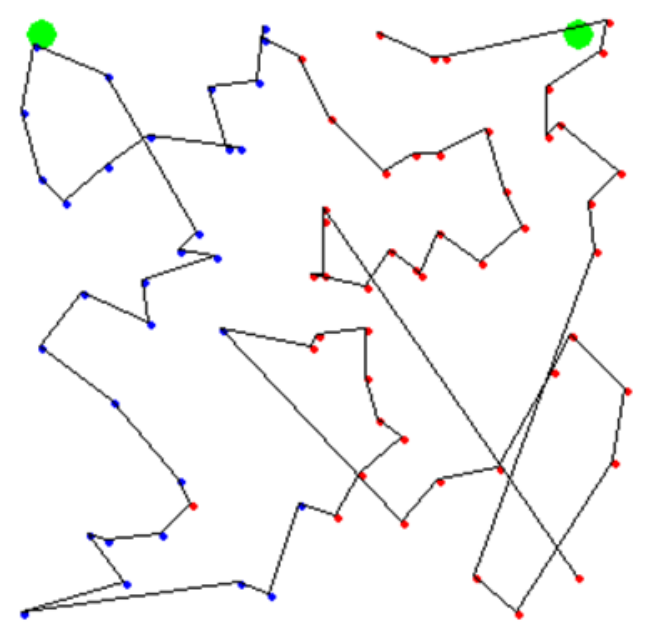

Fig. 6. Ruta de los Nodos

Como resumen de la solución de esta simulación se obtiene que al correr 100 veces el algoritmo la mejor ruta en este algoritmo dio como resultado un costo de $\$ 854$ donde la mejor solución se puede apreciar a continuación:

$\{062231244529173127593062434116371$ 1428527948182378681643615775201947 56696535262541461533559547276504522 3245838706667535177374603964781071 $21401342367349\}$

En cuanto al tema de asignación se tiene para cada depósito lo siguiente:

Depósito 1, ubicación $\{1,0\}$ : a este depósito se le asignan los primeros 33 nodos, los cuales se pueden encontrar en la Tabla 3.

Tabla 3. Nodos asignados al depósito 1

\begin{tabular}{|c|c|c|c|c|c|c|}
\hline 4 & 9 & 15 & 16 & 19 & 20 & 22 \\
\hline 25 & 26 & 32 & 33 & 35 & 38 & 41 \\
\hline 43 & 45 & 46 & 47 & 50 & 54 & 55 \\
\hline 56 & 57 & 58 & 61 & 64 & 65 & 69 \\
\hline 70 & 72 & 75 & 76 & 0 & & \\
\hline
\end{tabular}

Depósito 2, ubicación $\{90,0\}$ : este depósito tiene asignado desde el nodo 34 hasta el nodo 81; los nodos que se le asignaron a este depósito se encuentran en la Tabla 4.

Tabla 4. Nodos asignados al depósito 2

\begin{tabular}{|c|c|c|c|c|c|c|}
\hline 1 & 2 & 3 & 5 & 6 & 7 & 8 \\
\hline 10 & 11 & 12 & 13 & 14 & 17 & 18 \\
\hline 21 & 23 & 24 & 27 & 28 & 29 & 30 \\
\hline 31 & 34 & 36 & 37 & 39 & 40 & 42 \\
\hline 44 & 48 & 49 & 51 & 52 & 53 & 59 \\
\hline 60 & 62 & 63 & 66 & 67 & 68 & 71 \\
\hline 73 & 74 & 77 & 78 & 79 & & \\
\hline
\end{tabular}

\section{CONCLUSIONES}

Este tipo de algoritmos son una gran herramienta para asignar y rutiar la distribución de la mercancía, sin embargo es importante tener en cuenta que se deben analizar los resultados arrojados y adaptarlos a las necesidades, operación y recursos con los que cuenta la empresa.

Este algoritmo se fundamenta en la ruta y la distancia y no tiene en cuenta la capacidad de los depósitos en el momento de realizar la asignación.

El crecimiento que están teniendo las ciudades actualmente es lo que genera la búsqueda de modelos de distribución apoyados en buenas infraestructuras que les permite ser más eficientes en el sistema de distribución de la ciudad.

Se logró obtener una definición clara acerca de cada uno de los conceptos relacionados con el desarrollo de un modelo de distribución logística de mercancías.

\section{REFERENCIAS}

[1] S. Baeriswyl, "Plataforma Logística región del Bio Bio". Gobierno de Chile. 2005.

[2] R. Ballou, "Logística Empresarial Control y Planificación". Madrid: Díaz de Santos. 1991.

[3] E. A. Bermeo, J. H. Calderón, "Diseño de un modelo de optimización de rutas de transporte". El Hombre y la Maquina, (32), 52, 67. 2009.

[4] M. Browne. "Developments in urban distribution in London. Logistics Systems for Sustainable Cities: Proceedings of the 3rd International Conference on City Logistics". Madeira, Portugal. 2003.

[5] CITIVAS. (s.f.). "Logística y distribución de mercancías". Online [agosto, 2011].

[6] Concejo Privado de Competitividad. "Informe Nacional de Competitividad 2013-2014". 2013.

[7] Concejo Privado de Competitividad. "Informe Nacional de Competitividad 2012-2013". 2012.

[8] G. Ghiani, G. Laporte, and R. Musmanno. "Introduction to Logistics Systems Planning and Control". England: John Wiley \& Sons Ltd. 2004.

[9] M. González, N. Martínez, V. García, R. A. Eleuterio. "Estudio de Tres Algoritmos Heurísticos para Resolver un Problema de Distribución con Ventanas de Tiempo: Sistema por Colonia de Hormigas, Búsqueda Tabú y Heurístico Constructivo de una Ruta". 2009.

[10]J. Muñuzuri, J. Larrañeta, C. Muñoz, "La logística Urbana de Mercancías: Soluciones, Modelado y Evaluación. II Conferencia de Ingeniería de Organización". Vigo, España. 2002.

[11]A. Olivera, "Heurísticas para Problemas de Ruteo de Vehículos". Montevideo, Uruguay. 2004.

[12] J. A. Orjuela, O. F. Castro and E. A. Suspes, "Operadores y Plataformas Logísticas". España. Tecnura, vol. 8, No 16, 2005. 
[13] S. N. Parragh, K. F. Doerner, R. F. Hartl, "A survey on pickup and delivery problems. Part I: Transportation between customers and depot". Australia, 2008.

[14]C. Rodríguez, "Algoritmos heurísticos y metaheurísticos para el problema de localización de regeneradores". Proyecto fin de carrera. Universidad Rey Juan Carlos. Madrid, España. 2010.

[15] F. Sandoya, "Métodos Exactos y Heurísticos para resolver el Problema del Agente Viajero (TSP) y el
Problema de Ruteo de Vehículos (VRP)". Decimocuartas Jornadas en Estadística e informática. Guayaquil, Ecuador. 2010.

[16]E. Taniguchi, R. Thompson and T. Yamada, "Visions for city logistics. Logistics Systems for Sustainable Cities: Proceedings of the 3rd International Conference on City Logistics". Madeira, Portugal. 2001.

[17]J. A. Valles. "City Logistics y transporte de mercancías asistido con SIG". Reunión Nacional Usuarios SIGSA-Esri. 2011. 